\title{
Transmission datarate adap- tation using redundant check information for IEEE 802.11ax wireless LAN
}

\author{
Kazuto Yano ${ }^{1 a)}$, Kenta Suzuki ${ }^{1}$, \\ Babatunde Ojetunde $^{1}$, and Koji Yamamoto ${ }^{2}$ \\ ${ }^{1}$ Wave Engineering Laboratories, Advanced Telecommunications Research In- \\ stitute International \\ 2-2-2 Hikarida, Seika-cho, Soraku-gun, Kyoto 619-0288, Japan \\ ${ }^{2}$ Graduate School of Informatics, Kyoto University \\ Yoshida-honmachi, Sakyo-ku, Kyoto, Kyoto 606-8501, Japan \\ a)kzyano@atr.jp
}

\begin{abstract}
This letter proposes a practical transmission datarate adaptation (TDA) scheme using Q-learning applicable to IEEE 802.11ax wireless local area networks. In the proposed scheme, each basic service set (BSS) selects an appropriate transmission datarate according to the buffer statuses of adjacent BSSs which are periodically collected and the transmission results of DATA frames in the BSS. Then, the BSS conducts underlay transmissions based on the framework of spatial reuse defined in IEEE 802.11ax. The performance of the proposed scheme is evaluated through system-level computer simulation assuming downlink full-buffer traffic. It is confirmed that the proposed scheme can achieve higher average area throughput than conventional TDA schemes such as Robust Rate Adaptation Algorithm (RRAA) and adaptive modulation and coding (AMC) based on the received power. Keywords: wireless LAN, IEEE 802.11ax, Q-learning, transmission datarate adaptation, underlay transmission
\end{abstract}

Classification: Wireless communication technologies

\section{References}

[1] IEEE Std 802.11-2020, Feb. 2021.

[2] W. Yin, P. Hu, J. Indulska, M. Portmann, and Y. Mao, "MAC-layer rate control for 802.11 networks: Lesson learned and looking forward," arXiv:1807.02827v1, July 2018.

[3] A. Karmerman and L. Monteban, "WaveLAN-II: A high-performance wireless LAN for the unlicensed band," Bell Labs Technical Journal, vol. 2, no. 3, pp. 118-133, 1997. DOI:10.1002/bltj.2069

[4] S. H. Y. Wong, H. Yang, S. Lu, and V. Bharghavan, "Robust rate adaptation for 802.11 wireless networks," Proc. ACM MOBICOM'06, pp. 146157, September 2006. DOI:10.1145/1161089.1161107

[5] H. Senda, O. Takyu, A. Kamio, M. Ohta, and T. Fujii, "Discrimination of communication quality deterioration utilizing retransmission flag and 
[6] K. Yamamoto, M. Mieda, S. Kondo, T. Nishio, A. Taya, and K. Yano, "Interference source determination based on history of transmissions in WLANs," IEICE Tech. Rep., RCS2021-141, pp. 122-125, October 2021.

[7] K. Yamamoto, Y. Kihira, Y. Koda, T. Nishio, and M. Morikura, "Factor analysis of communication quality using redundancy-check information in wireless LANs," Proc. IEICE Gen. Conf 2020, B-5-147, March 2020.

[8] IEEE Std 802.11ax-2021, May 2021.

[9] R. Porat, et al, "11ax Evaluation Methodology," doc. IEEE 802.1114/0571r12, January 2016.

\section{Introduction}

IEEE 802.11 wireless local area networks (WLANs) [1] have been widely and densely deployed, and their demand is still growing. The current WLAN generally employs a distributed and autonomous channel access mechanism based on carrier sense multiple access with collision avoidance (CSMA/CA). Therefore, increase of traffic demand in the WLAN brings severe contention among multiple basic service sets (BSSs) sharing the same radio channel. It causes frequent collision and resultant failure of frame transmission.

Since IEEE 802.11 WLANs support multiple transmission datarates, each transmitter needs to adjust its transmission datarate to a suitable one. When the signal-to-noise ratio (SNR) of a transmitted frame is insufficient, its transmitter should lower the transmission datarate. On the other hand, when transmission failure is caused by collision, the transmission datarate should be raised so as to shorten the length of the transmitted frame and to reduce the probability of collision consequently.

Therefore, in general, the transmission datarate is adjusted according to one or more metrics calculated from the results of frame transmission [2]. For example, Automatic Rate Fallback (ARF) [3] raises/decreases the transmission datarate if the number of successful/failed frame transmissions reaches pre-determined a threshold. Robust Rate Adaptation Algorithm (RRAA) [4] raises/decreases the transmission datarate if a frame error rate (FER) becomes larger/less than a given FER threshold.

However, IEEE 802.11 WLANs have no way to know directly whether a failure of frame transmission is caused by collision or by other reasons such as insufficient SNR because the transmitter recognizes its transmission failure by occurring a timeout of ACK frame reception. Several studies were recently conducted to estimate the factor of transmission failure using one or more frame sniffers to utilize the estimated cause to adjust the transmission datarate $[5,6]$.

Furthermore, a transmission datarate adaptation (TDA) scheme was studied to selects an appropriate transmission datarate using Q-leaning with the aid of side information called "redundant check information" about the frame transmission in adjacent basic service sets (BSSs) [7]. This scheme collects, as the redundant check information, the information whether or not adjacent 
BSSs will transmit their frames in near future, and then learns and selects the best action (i.e., selects the best transmission datarate or defers its frame transmission). If the best transmission datarate is selected, each BSS makes underlay transmission against its adjacent BSSs, and thus this scheme can improve the throughput.

However, the performance evaluation conducted in [7] assumes slotted channel access. On the other hand, IEEE 802.11 WLAN employs random backoff based on CSMA/CA [1], and thus it is difficult to precisely know when each node will transmit its frame. Hence, this letter proposes a practical scheme to apply the concept of transmission datarate adaptation (TDA) in [7] to IEEE 802.11ax [8] which defines a mechanism of spatial reuse for underlay transmissions. The proposed scheme adjusts the transmission datarate of each BSS based on Q-learning at an adaptation interval. The buffer status of each BSS is collected as the redundant check information, and it is obtained using buffer status report (BSR) defined in IEEE 802.11ax.

The remainder of this paper is as follows. Section 2 introduces the concept of the TDA presented in [7] and our proposed TDA scheme. Section 3 explains the configuration of the system-level computer simulation and its results. Finally, conclusion is given in Sect. 4.

\section{TDA using redundant check information}

Figure 1(a) shows the the concept of the TDA presented in [7] applicable to slotted channel access. We focus on BSS 0 as a target BSS of TDA, and other BSSs are adjacent BSSs. BSS 0 collects the information whether adjacent BSSs will transmit their frames or not in the next slot. As shown in Fig. 1(a), this information is encoded as "state." BSS 0 selects the transmission datarate with the maximum Q-value on the state, or selects a transmission datarate randomly with a certain probability. (Hereafter, this probability is called as "random selection probability.") BSS 0 makes its DATA frame transmission at the selected transmission datarate, and then updates the Q-value according to the result of frame transmission. This process is conducted slot-by-slot (in other words, frame-by-frame). This scheme can improves throughput comparing with the conventional slotted ALOHA because it can select an appropriate transmission datarate even when collision is expected.

Figure 1(b) shows the concept of our proposed TDA scheme. In our proposed scheme, the transmission datarate is adjusted at an interval $\left(T_{\mathrm{c}}\right)$. Since BSS 0 cannot know when adjacent BSSs will transmit their frames exactly due to the random backoff, it collects the buffer statuses of adjacent BSSs, and selects the transmission datarate used in the next adaptation period. Here, an access point (AP) in each BSS can obtain the buffer status of its associating stations (STAs) by BSR. Each node checks by using BSS Color [8] defined in IEEE 802.11ax whether the received frame comes from the BSS that is expected to transmit frame(s) in the current adaptation period. (The state of such BSS is denoted by "1" in Fig. 1(b).) If the node detects a 


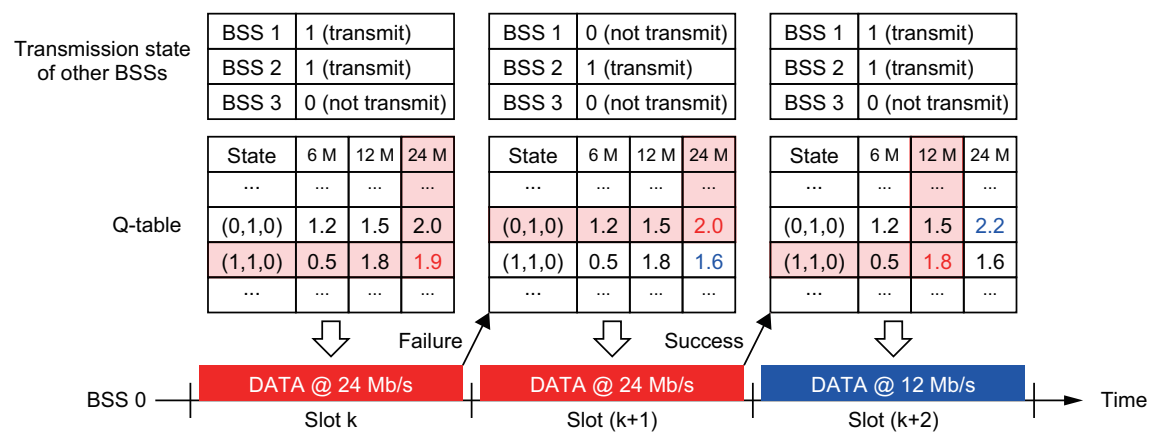

(a) Concept of TDA presented in [7].

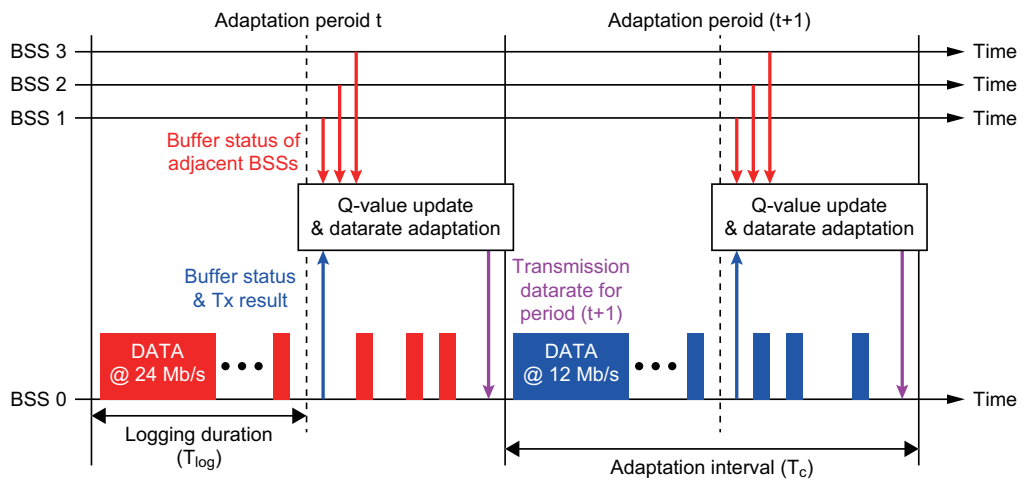

(b) Concept of the proposed TDA.

Fig. 1. Concept of TDA using redundant check information and Q-learning.

frame from such BSS, the CCA level at the node is raised so that the frame from the BSS is not detected using the framework of spatial reuse, which is also defined in IEEE 802.11ax, in order to enable underlay transmissions.

The Q-value is updated by the transmission results of DATA frames in a logging duration ( $T_{\log }$ from the beginning of the adaptation period) frameby-frame as follows.

$$
Q\left(s_{t}, a_{t}\right) \leftarrow Q\left(s_{t}, a_{t}\right)+\alpha\left\{r_{t+1}-Q\left(s_{t}, a_{t}\right)\right\}
$$

where $\alpha$ is the learning rate, $s_{t}$ is the state of buffers in adjacent BSSs, and $a_{t}$ is the selected action of the target BSS (i.e., BSS 0 in Fig. 1(b)). The reward $r_{t+1}$ of each DATA frame is calculated by

$$
r_{t+1}=\left\{\begin{array}{l}
\left(\delta_{X, 1} W_{\mathrm{suc}}-\delta_{X, 0} W_{\text {fail }}\right) R D \quad \text { if } \quad R \geq 0 \\
r_{\text {notx }} \text { if } \quad R=-1
\end{array}\right.
$$

where $\delta_{a, b}$ denotes Kronecker delta, $X$ is the transmission result (" 1 " means SUCCESS, and "0" means FAILURE) of the DATA frame, $D$ [kbyte] is the payload length of the DATA frame, $W_{\text {suc }}$ and $W_{\text {fail }}$ are the weights of reward for successful and failed frame transmissions, respectively. $R$ is the used transmission datarate $[\mathrm{Mb} / \mathrm{s}]$. Here, $R=-1$ denotes that frame transmission 
is pended in the corresponding adaptation period, and the reward is set to $r_{\text {notx }}$ in this case.

\section{Computer simulation}

The performance of the proposed TDA scheme is evaluated through systemlevel computer simulation based on IEEE 802.11ax WLAN. We compare three schemes: our proposed scheme, RRAA, and adaptive modulation and coding (AMC) based on the received power.

Table I shows the simulation parameters. The area size is assumed to be $80 \mathrm{~m} \times 80 \mathrm{~m}$, and it is segmented into $4 \times 4$ (thus, the segment size is $20 \times$ $20 \mathrm{~m})$. Each BSS is located in different segment. In our proposed scheme, each BSS collects the buffer statuses of the BSSs whose frame is received with the received power equal to or greater than $-88 \mathrm{dBm}$ which is same as the frame detection limit in this simulation. The random selection probability of action $P_{\text {rand }}$ at the $t$ th adaptation period is set to $P_{\text {rand }}=1 /(1+t / N)$, and $N$ is set to 133 , which gives $P_{\text {rand }}=0.1$ at 2 minutes, because it gives high performance in the preliminary simulation.

In RRAA, the transmission datarate is adjusted every $T_{\mathrm{c}}$ if the number of transmitted DATA frames after the previous datarate adjustment is equal to or greater than 50. If frame error rate (FER) is greater than 0.1, the modulation and coding scheme (MCS) index is decremented by one, and it is incremented by one if the FER is less than 0.05. Otherwise, the current MCS index is retained. In AMC, the maximum MCS index at which the received power satisfies the minimum input sensitivity defined in IEEE 802.11ax [8].

Table I. Simulation parameters

\begin{tabular}{l|l}
\hline \hline Evaluation duration & 20 minutes \\
\hline Number of BSSs & 4,16 \\
\hline Number of STAs per BSS & 1 \\
\hline Supported MCS index & $0-9$ for IEEE $802.11 \mathrm{ax}[8]$ \\
\hline Transmission power & $20 \mathrm{dBm}$ \\
\hline Signal bandwidth & $20 \mathrm{MHz}$ \\
\hline Noise level & $-94 \mathrm{dBm}$ (including $7 \mathrm{~dB}$ noise figure $)$ \\
\hline Propagation model & IEEE 802.11 TGax Residential scenario $[9]$ \\
\hline Frequency channel & Ch 1 in the $2.4 \mathrm{GHz}$ band \\
\hline Retransmission limit & 7 times \\
\hline RTS/CTS exchange & Not in use \\
\hline Traffic & Downlink full-buffer traffic \\
\hline Payload length & 3000 bytes \\
\hline Interval of datarate adaptation $\left(T_{\mathrm{c}}\right)$ & $100 \mathrm{~ms}$ \\
\hline Logging duration $\left(T_{\text {log }}\right)$ & $80 \mathrm{~ms}$ \\
\hline Leaning rate $(\alpha)$ & 0.1 \\
\hline Weights of reward & $\left(W_{\text {suc }}, W_{\text {fail }}\right)=(1,0.1)$ \\
\hline Reward for pending transmission $\left(r_{\text {notx }}\right)$ & -2 \\
\hline \hline
\end{tabular}

Figures $\mathbf{2}(\mathrm{a})$ and $\mathbf{2}(\mathrm{b})$ shows the average area throughput and the frame delivery rate of AMC, RRAA and the proposed scheme, respectively. In addition, the number of DATA frames transmitted at each MCS index when the number of BSSs is 16 is shown in Fig. 2(c). The proposed scheme achieves 
the area throughput 1.3 and 2.1 times (1.4 and 4.0 times) as high as that of AMC and RRAA when the number of BSSs is 4 (16), respectively. AMC can achieve higher throughput than RRAA even though its frame delivery rate is worse than RRAA because AMC uses much higher MCS index (in other words, higher transmission datarate) than RRAA. On the other hand, the proposed scheme achieves a frame delivery rate almost as high as RRAA, and it tends to use higher MCS index whereas RRAA tends to use lower MCS index. It means the proposed scheme can select an appropriate MCS index according to the buffer statuses of adjacent BSSs.

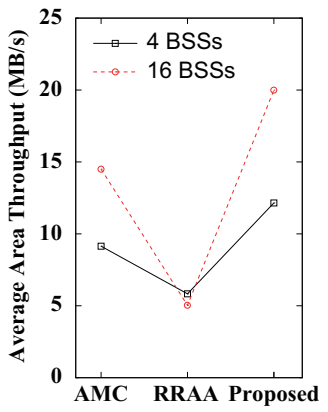

(a) Average throughput.

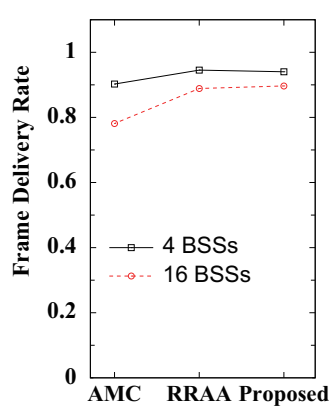

(b) Frame delivery rate.

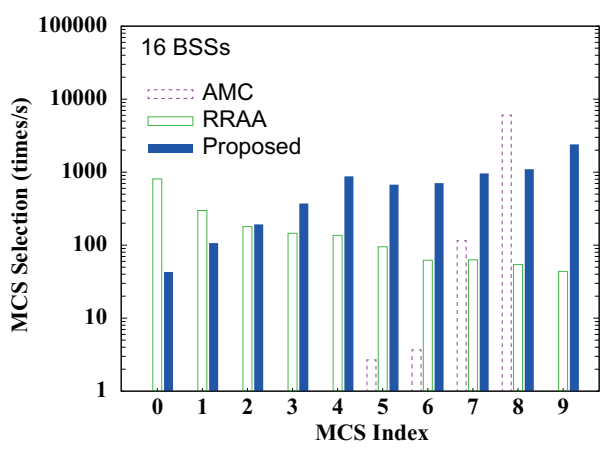

(c) Number of DATA frame transmissions (16 BSSs).

Fig. 2. Simulation results.

\section{Conclusion}

This letter proposed a practical TDA scheme using Q-learning applicable to IEEE 802.11ax WLAN. In the proposed scheme, each BSS selects an appropriate transmission datarate according to the buffer statuses of adjacent BSSs which are periodically collected and the transmission results of DATA frames in the BSS. Then, the BSS conducts underlay transmissions based on the framework of spatial reuse defined in IEEE 802.11ax. The performance of the proposed scheme was compared with the conventional TDA schemes: RRAA and AMC based on the received power through system-level computer simulation based on IEEE 802.11ax WLAN assuming downlink fullbuffer traffic. It was confirmed that the proposed scheme achieves the area throughput 1.3 times and 2.1 times (1.4 and 4.0 times) as high as that of AMC and RRAA when the number of BSSs sharing the same frequency channel is 4 (16), respectively, by selecting a proper MCS index according to the buffer statuses of adjacent BSSs.

\section{Acknowledgments}

This research and development work was supported by the MIC/SCOPE \#JP196000002. 